\title{
Successful Treatment of Spontaneous Rupture of the Spleen by Embolization of Splenic Artery in a Patient with Acute Promyelocytic Leukaemia and COVID-19 Infection
}

\author{
Olivera Markovic ${ }^{1,2}$, Anica Divac ${ }^{*}$, Filip Lukic ${ }^{3}$, Davor Mrda3 ${ }^{3}$, Ana Vidovic ${ }^{2,4}$, \\ Marija Zdravkovic ${ }^{2,5}$, Borislav Toskovic ${ }^{2,6}$ \\ ${ }^{1}$ Department of Haematology, Clinical Hospital Centre “Bezanijska Kosa”, Belgrade, Serbia \\ ${ }^{2}$ Faculty of Medicine, Belgrade, Serbia \\ ${ }^{3}$ Department of Radiology, Clinical Hospital Centre "Bezanijska Kosa”, Belgrade, Serbia \\ ${ }^{4}$ Clinic of Haematology, Clinical Centre of Serbia, Belgrade, Serbia \\ ${ }^{5}$ Department of Cardiology, Clinical Hospital Centre "Bezanijska Kosa”, Belgrade, Serbia \\ ${ }^{6}$ Department of Surgery, Clinical Hospital Centre “Bezanijska Kosa”, Belgrade, Serbia \\ Email: *markovic.olivera@bkosa.edu.rs, *anicadivac92@gmail.com
}

How to cite this paper: Markovic, O., Divac, A., Lukic, F., Mrda, D., Vidovic, A., Zdravkovic, M. and Toskovic, B. (2021) Successful Treatment of Spontaneous Rupture of the Spleen by Embolization of Splenic Artery in a Patient with Acute Promyelocytic Leukaemia and COVID-19 Infection. Open Journal of Emergency Medicine, 9, 60-65.

https://doi.org/10.4236/ojem.2021.93008

Received: May 30, 2021

Accepted: July 3, 2021

Published: July 6, 2021

Copyright $\odot 2021$ by author(s) and Scientific Research Publishing Inc. This work is licensed under the Creative Commons Attribution International License (CC BY 4.0)

http://creativecommons.org/licenses/by/4.0/

\begin{abstract}
Spontaneous rupture of the spleen (SRS) is a rare clinical entity with a potentially poor medical outcome. In most cases, SRS is caused by neoplastic disorder. Acute promyelocytic leukaemia is a rare but important cause of SRS that physicians are required to assess for. We present a 28 -year-old woman with APL and COVID-19 pneumonia, who successfully underwent embolisation of the splenic artery for spontaneously occurring splenic rupture during induction chemotherapy. After the intervention the patient completed induction chemotherapy and achieved complete remission. Our case demonstrates that emergent transcatheter arterial embolisation can be lifesaving even in the unfavourable condition of a patient with severe immune deficiency.
\end{abstract}

\section{Keywords}

Acute Promyelocytic Leukaemia, COVID-19, Embolisation of Splenic Artery, Splenic Rupture

\section{Introduction}

Spontaneous rupture of the spleen (SRS) is a rare clinical entity with a potentially poor medical outcome. It may be caused by neoplastic, infectious and inflammatory diseases and mechanical disorders or may be drug and treatment related. 
Among hematological malignancies, SRS most often occurs in patients with acute leukaemia, chronic myeloid leukaemia and non-Hodgkin lymphoma [1]. Acute promyelocytic leukaemia (APL) is a unique subtype of acute myeloid leukaemia (AML), cytogenetically characterized by a balanced reciprocal translocation between chromosomes 15 and 17, which results in the fusion of the promyelocytic leukaemia (PML) and retinoic acid receptor alpha (RAR $\alpha$ ) genes [2]. The most prominent clinical feature of APL is a rare severe haemorrhagic diathesis [2]. However, SRS is an extremely rare complication in APL. To date, only 3 cases of APL with SRS treated with splenectomy have been reported in literature [3] [4] [5]. The SRS is a complication that requires immediate diagnosis and prompt surgical treatment [3]. Splenectomy is recommended as a treatment choice in haemodynamic unstable patients, but in APL with coagulation disorder and immunity impairment, these patients have a high risk of mortality. We present the first case of APL and SRS successfully treated by transcatheter arterial embolisation (TAE) of the splenic artery and give an overview of similar cases reported in literature.

\section{Presentation of the Case}

A 28-year-old female patient was transferred to our Department of Haematology from a non-COVID-19 Haematology Clinic due to infection with COVID-19 and treatment of APL. Over a period of 10 days before admission, the patient complained of fatigue, spontaneous haematomas and febrile episodes. Diagnosis of APL was established in another Haematology Clinic. The initial blood count and laboratory evaluation showed anaemia at $104 \mathrm{~g} / \mathrm{l}$, severe thrombocytopenia at $9 \times 10^{9} /$, leucocytosis at $22.1 \times 10^{9}$ and pronounced coagulopathy (fibrinogen 1.2 g/l, PT 58\%, aPTT 35.7 s D-dimer 34,000 ng/ml). Serum lactate dehydrogenase was elevated $(2272 \mathrm{U} / \mathrm{l})$. The COVID-19 PCR test was negative (sample taken $72 \mathrm{~h}$ before admission). A chest X-ray showed signs of left-sided incipient pneumonia. Abdominal ultrasound showed hepatic and splenic enlargement. Bone marrow aspiration showed infiltration of $88 \%$ POX positive blast cells with hypogranularpromyelocytes. Conventional bone marrow cytogenetic analysis revealed the presence of $t(15,17)$. RT-PCR showed the presence of the PML-RAR- $a$ fusion gene. Treatment according to the Spanish PETHEMA 2012 protocol was initiated and the patient received therapy scheduled for the first 2 days. However, a subsequent COVID-19 PCR test taken on admission was positive. As infection with COVID-19 was confirmed, the patient was transferred to our Department of Haematology intended for treatment of haematological patients with COVID-19. The treatment of COVID-19 infection was initiated according to the national protocol, and induction chemotherapy for APL was continued. On the ninth day of chemotherapy, the patient complained of sudden severe pain below the left costal margin. On physical examination, the patient was pale, diaphoretic, normotensive, tachycardic (101 beats/min), the abdomen was painful with hepatosplenomegaly. The blood count showed a reduction of haemoglobin levels from 110 to $96 \mathrm{~g} / \mathrm{l}$. Computerised tomographic (CT) of the abdomen which had 
been urgently done showed an enlarged liver $(230 \mathrm{~mm})$ and spleen $(220 \mathrm{~mm})$ with slow post-contrast opacification and intraparenchymal splenic rupture (Figure 1).

A surgical and radiological consultation was obtained and TAE of the splenic artery as a lifesaving emergency intervention was done after the preparation of the patient with transfusion of cryoprecipitates, fresh frozen plasma, packed red cells and platelets. Catheterisation showed an intraparenchymal rupture of the spleen (Figure 2).

The procedure is performed using a local anesthetic and puncture of a right femoral artery according to the Seldinger method and after that through transcatheter approach to splenic artery. The intervention was performed with 3 detachable coils $(14 \mathrm{~mm} \times 30 \mathrm{~cm})$ and 3 pushable coils $(10 \mathrm{~mm} \times 10 \mathrm{~cm})$ which resulted in "near to statis" arterial occlusion (Figure 2). A few hours after the intervention the abdominal pain had disappeared and the patient was haemodynamically stable. The follow-up CT scan which was done after 2 weeks revealed that the splenic haemorrhage had been controlled by embolization. However, there was no reduction in liver and spleen size. The patient completed induction chemotherapy in our department. The parameters of blood count and coagulation before and after intervention are showed in Table 1. As a control CT scan of the chest showed regression of pneumonia and the PCR test for COVID-19 came in negative two times, the patient was transferred to a COVID-19-free Clinic. At this point, the patient was receiving consolidation therapy.

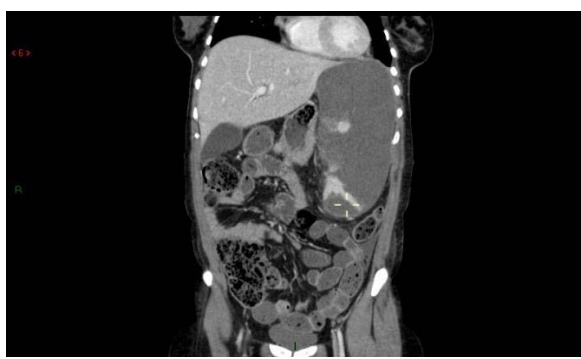

Figure 1. Initial CT scan of abdomen.

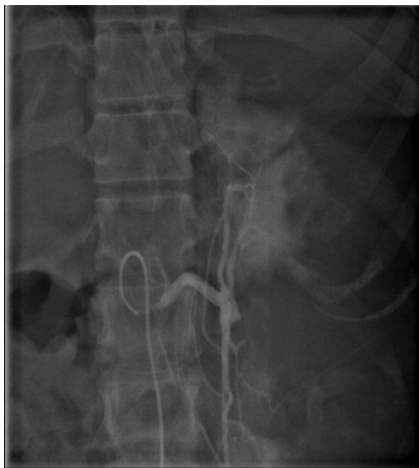

(a)

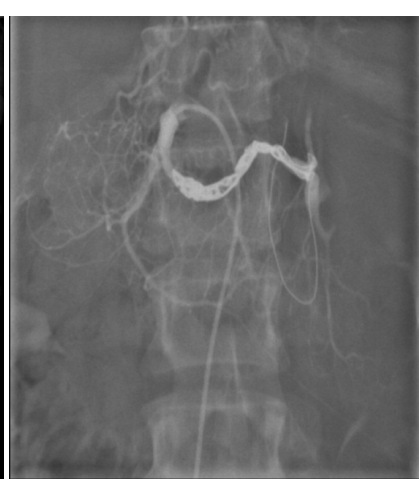

(b)

Figure 2. (a) Angiography of the splenic artery showing contrast extravasation and blurring in the spleen; (b) Emergency TAE using detachable and pushable coils resulted in a successful control of haemorrhage. 
Table 1. Parameters before and after intervention.

\begin{tabular}{ccc}
\hline Leucocytes $\left(\times 10^{9} / \mathrm{l}\right)$ & 22.3 & 6.83 \\
\hline Haemoglobin $(\mathrm{g} / \mathrm{l})$ & 96 & 94 \\
Platelets $\left(\times 10^{9} / \mathrm{l}\right)$ & 5 & 350 \\
Fibrinogen $(\mathrm{g} / \mathrm{l})$ & 1.1 & 3.9 \\
$\operatorname{aPTT}(\mathrm{s})$ & 33.9 & 20 \\
D-dimer $(\mathrm{ng} / \mathrm{ml})$ & 33,400 & 2027 \\
\hline
\end{tabular}

\section{Disscusion}

SRS is a rare complication of different haematological malignancies associated with high mortality [1]. The most common manifestations of SRS are signs of intraabdominal bleeding. Considering the nonspecificity of signs and symptoms and the rarity of SRS, diagnosis and management may be delayed. According to a previous report the diagnosis of SRS was made in almost half of the cases only after laparotomy [1] [6]. In case of delay in diagnosis the prognosis is worse and the mortality rate increases $(1,12)$. Increased mortality is also associated with massive splenomegaly, older age and advanced malignant diseases [1] [7].

The management of all haematological malignancies in the context of COVID-19 poses a challenge for clinicians especially in cases of alife-threatening complication such as SRS. The management of APL in association with COVID-19 poses an even greater challenge because both diseases are associated with severe coagulopathy [2] [8] [9]. Previously, splenectomy was considered the therapy of choice in patients with SRS. However, early TAE of the splenic artery has recently become the preferred choice in patients with blunt splenic trauma and in patients with hypersplenism, given its promising results and decreased morbidity, especially in patients who are at high risk for splenectomy [10] [11]. It is unknown what the optimal therapeutic approach in patients with a haematological disorder and SRSis, especially in the context of pre-existing haemorrhagic diathesis and COVID-19 infection. In three published cases with APL and most cases with other types of acute leukaemia, patients were successfully treated with an immediate splenectomy [1]. Some literature research revealed that TAE of the splenic artery was performed in a few patients with haematological malignancies [7] [12] [13] [14]. Considering the poor coagulation status of the patient, the expected bone marrow aplasia after induction therapy and COVID-19 infection, splenectomy was estimated as a high-risk intervention in our patient. Therefore, we decided to do TAE of the splenic artery which was done successfully.

\section{Conclusion}

SRS is a rare complication in APL with a high rate of mortality. Splenectomy in patients with APL is a high-risk intervention due to coagulation disorder, decreased count of platelets and impaired immunity. Rapid diagnosis and early treatment are necessary for survival of these patients. A favourable outcome in 
our patient suggests that TAE of splenic artery should be considered in patients with haematological malignancies who experience SRS during chemotherapy, especially in patients who are at high risk for surgical intervention and in patients where the diagnosis is made before the development of hemodynamic instability.

\section{Conflicts of Interest}

The authors declare no conflicts of interest regarding the publication of this paper.

\section{References}

[1] Renzulli, P., Hostettler, A. and Schoepfer, A.M. (2009) Systematic Review of Atraumatic Splenic Rup Ture. British Journal of Surgery, 96, 1114-1121.

https://doi.org/10.1002/bjs.6737

[2] Thomas, X. (2019) Acute PromyelocyticLeukemia: A History over 60 Years-From the Most Malignant to the Most Curable Form of Acute Leukemia. Oncology and Therapy, 7, 33-65. https://doi.org/10.1007/s40487-018-0091-5

[3] Hernández, R., del Cañizo, M.C., López, C., González, M.I., Vázquez, M.L., Caballero, M.D. and Miguel, J.F.S. (2000) Pathologic Rupture of the Spleen during Induction with ATRA in a Patient with Acute Promyelocytic Leukemia. Medical Oncology, 17, 337-339. https://doi.org/10.1007/BF02782201

[4] Kuba, A., Szotkowski, T. and Rohon, P. (2015) Spontaneous Splenic Rupture in a Patient with Acute Promyelocytic Leukaemia during Induction Chemotherapy. Biomedical papers of the Medical Faculty of the University Palacky, Olomouc, Czechoslovakia, 159, 294-298. https://doi.org/10.5507/bp.2013.086

[5] Lazzarino, M., Morra, E. and Castagnola, C. (1990) Spontaneous Splenic Rupture during Induction Chemotherapy for Acute Promyelocytic Leukemia Successfully Treated with Splenectomy. Haematologica, 75, 84-86.

[6] Bauer, T.W., Haskins, G.E. and Armitage, J.O. (1981) Splenic Rupture in Patients with Hematologic Malignancies. Cancer, 48, 2729-2733. https://doi.org/10.1002/1097-0142(19811215)48:12<2729::AID-CNCR2820481229> 3.0.CO;2-K

[7] Wu, M.Y., Kao, W.Y., Chan, C.Y., Yiang, G.T., Liao, W.T. and Chen, C.S. (2019) Spontaneous Splenic Rupture as a Rare Initial Presentation in an Acute Lymphoblastic Leukemia Patient. Diagnostics, 9, 152. https://doi.org/10.3390/diagnostics9040152

[8] Bikdeli, B., Madhavan, M.V., Jimenez, D., Chuich, T., Dreyfus, I. and Driggin, E. (2020) COVID-19 and Thrombotic or Thromboembolic Disease: Implications for Prevention, Antithrombotic Therapy, and Follow-Up: JACC State-of-the-Art Review. Journal of the American College of Cardiology, 75, 2950-2973. https://doi.org/10.1016/j.jacc.2020.04.031

[9] Baldacini, M., Pop, R., Sattler, L., Mauvieux, L., Bilger, K., Gantzer, J., Schneider, F. Beaujeux, R., Simand, C. and Herbrecht, R. (2020) Concomitant Haemorrhagic Syndrome and Recurrent Extensive Arterial Thrombosis in a Patient with COVID-19 and Acute Promyelocytic Leukaemia. British Journal of Haematology, 189, 1054-1056. https://doi.org/10.1111/bjh.16768

[10] Wahl, W.L., Ahrns, K.S. and Chen, S. (2004) Blunt Splenic Injury: Operation versus Angiographic Embolization. Surgery, 136, 891-899. 
https://doi.org/10.1016/j.surg.2004.06.026

[11] Ahuja, C., Farsad, K. and Chadha, M. (2015) Overview of Splenic Embolization. American Journal of Roentgenology, 205, 720-725.

https://doi.org/10.2214/AJR.15.14637

[12] Jafferbhoy, S., Chantry, A., Atkey, N., et al. (2011) Spontaneous Splenic Rupture: An Unusual Presentation of CML. BMJ Case Reports, 2011, bcr0220113879. https://doi.org/10.1136/bcr.02.2011.3879

[13] Sachie, W., Koichi, K., Takayuki, S., Akira, F. and Seiichiro, S. (2013) Splenic Rupture Associated with Aggressive Conversion of Indolent T-Cell Prolymphocytic Leukemia. Rinsho Ketsueki, 54, 284-289.

[14] Gardner, R.V., Warrier, R.P., Loe, W., Ward, K., Haymon, M. and Craver, R. (2003) Splenic Artery Embolization as Emergency Treatment of Splenic Rupture in a Child with T-Cell Acute Lymphocytic Leukemia Having t (8; 14) Translocation. Medical and Pediatric Oncology, 41, 492-493. https://doi.org/10.1002/mpo.10372 